\title{
Follow-up endoscopy for benign-appearing gastric ulcers has no additive value in detecting malignancy
}

\author{
It is time to individualise surveillance endoscopy
}

\author{
Eric A. R. Gielisse • Johan P. Kuyvenhoven
}

Received: 21 May 2014/ Accepted: 16 September 2014/Published online: 14 October 2014

(C) The International Gastric Cancer Association and The Japanese Gastric Cancer Association 2014

\begin{abstract}
Objective To determine the diagnostic accuracy of endoscopic follow-up for gastric ulcers.

Methods All cases of gastric ulcers diagnosed at our teaching hospital between September 2005 and November 2011 were reviewed. The cases were selected by using ENDOBASE, an endoscopy documentation system. The characteristics of the ulcers and their histology were analysed.

Results During the study period 321 cases with a gastric ulcer were diagnosed, including 214 benign ulcers $(67 \%)$ and 107 malignant ulcers $(33 \%)$. The mean age of the population was 71 years. In 200 patients $(62 \%)$ the ulcers were classified as benign appearing at the first endoscopy. However, in five of these patients, the ulcers eventually were malignant. In all of these five patients the index gastroscopy revealed a non-benign histology. Therefore, the sensitivity of a benign appearance of the ulcer in combination with histology at the first endoscopy is $100 \%$ to rule out malignancy. In 121 patients (38\%) the ulcers were explicitly labelled as potentially malignant in the report of the first endoscopy. Of these potentially malignant-appearing ulcers, $102(84 \%)$ were indeed malignant as confirmed by histology. The other 19 ulcers $(16 \%)$ were benign at follow-up. The sensitivity of the three potential malignant characteristics at endoscopy was: dirty base
\end{abstract}

E. A. R. Gielisse $(\bowtie)$

Department of Gastroenterology and Hepatology, VU University

Medical Center, P.O. Box 7057, 1007 MB Amsterdam,

The Netherlands

e-mail: ericgielisse@hotmail.com

J. P. Kuyvenhoven

Department of Gastroenterology and Hepatology, Kennemer

Gasthuis, Haarlem, The Netherlands
$79 \%$, elevated border $94 \%$ and irregular border $91 \%$. The specificity was 93,82 and $89 \%$, respectively. The median diameter of the ulcers was significantly higher in the malignant group compared to the benign ulcer group $(p<0.0001)$. The accuracy of endoscopic malignancy diagnosis was as follows: sensitivity of 0.98 and specificity 0.84 , positive predictive value 0.84 and negative predictive value 0.98 . In total, 546 gastroscopies were performed in these 321 patients, of which 225 were follow-up endoscopies. By not monitoring ulcers considered benign in both appearance and histology, 173 gastroscopies would not have been performed, resulting in a decline of $77 \%$ of the follow-up endoscopies performed.

Conclusion Endoscopic follow-up of gastric ulcers considered benign by appearance and with benign histology showed no additive value in detecting unsuspected malignancy in this study. This strategy could reduce health costs and save distress to patients.

Keywords Follow-up endoscopy · Gastric ulcer · Gastric cancer · Gastroscopy

\section{Introduction}

Gastroenterologists often encounter gastric ulcers. The yearly incidence is more than 5 per 1,000 adults [1]. Gastric ulcers are associated with the use of ulcerogenic drugs such as non-steroidal anti-inflammatory drugs (NSAIDs) and aspirin. Other important causes of gastric ulcers are Helicobacter pylori infection and malignancy.

Current European guidelines [2-4] are based on the publications in the late 1970s and early 1980s [5-8] and advise follow-up endoscopy for all gastric ulcers until healing is confirmed in order to exclude malignancy. Also, 
in the USA and Canada routine follow-up for gastric ulcers is a common practice.

Whether all gastric ulcers should be surveyed until healing is still being discussed [9-13]. There are also contradictory results about the improvement of survival secondary to an endoscopic follow-up strategy [13, 14]. An analysis of a cohort of 60-year-old males with presumed benign ulcers in the USA showed that follow-up surveillance increased life expectancy by only 10 days. This same study stated that surveillance is unlikely to be cost effective unless the prevalence of gastric cancer exceeds $6 \%$ [14].

Previous studies reported that $2-5 \%$ of the benignappearing ulcers are ultimately malignant [15]. The overall endoscopic and biopsy accuracy rate is reported as high as $99 \%[16,17]$.

The aim of this study was to determine the diagnostic accuracy of endoscopic follow-up of patients with a gastric ulcer with emphasis on endoscopic hallmarks of malignancy and histology at the first endoscopy.

\section{Patients and methods}

All cases of gastric ulcers diagnosed at our teaching hospital in The Netherlands between September 2005 and November 2011 were reviewed. The cases were selected by using ENDOBASE, searching for the terms 'gastric ulcer' and 'gastric tumour'. ENDOBASE is a documentation system that captures endoscopy information including the reports and pictures. Whether the endoscopic report contained an explicit suspicion of malignancy was noted. A rigid definition of a malignant appearance was not applied but all endoscopists used common criteria. Three specific potential malignant characteristics of the ulcer(s), i.e. a dirty base, elevated or irregular border of the ulcer, were separately reviewed. The base was considered 'dirty' if areas with necrosis and irregularity of different colours were present. The border was considered 'elevated' if raised in proportion to the centre of the base and the mucosa surrounding the ulcer. The characteristic 'irregular' was given to asymmetrical borders. Examples of benign and malignant ulcers are shown in Fig. 1.

Appearance as described in the report was evaluated in combination with histology of the first endoscopy. The size of the ulcers and their localisation in the stomach were registered as well. Finally, we registered ulcerative medication use and Helicobacter pylori infection. All endoscopies were performed by experienced board-certified gastroenterologists using conventional white light imaging (C-WLI) only. The endoscopies were performed for a wide range of indications of which nausea, vomiting and anaemia were the most prevalent.
The hospital policy was to follow up every ulcer endoscopically until healing had occurred. Biopsies of the scar after complete healing were not taken in a standardised way but individualised. The cases that were not followed up endoscopically until healing were only considered benign if the patients presented at our hospital a year or longer after the latest gastroscopy without specific gastrointestinal complaints and did not need to undergo a new gastroscopy. In case patients were not present at our hospital after the last gastroscopy the national pathology database (PALGA) was consulted to exclude gastric malignancy diagnosed in another hospital.

We excluded patients in whom healing of the ulcer was not confirmed endoscopically and who died within 1 year after index endoscopy.

Proportional differences were tested for statistical significance with the $\chi 2$ test or Fisher's exact test.

\section{Results}

During the 6-year period 341 patients with gastric ulcers were diagnosed. We excluded 20 cases because they were not followed up until healing and the patients died within a year after the initial endoscopy, thereby making the probability of excluding malignancy too low. Of the 321 patients included in this study, 214 were finally benign and in 107 patients the ulcers were proven malignant histologically. The demographic features of the two groups are shown in Table 1.

The classification of a benign ulcer was based on followup endoscopy until complete healing had occurred in 149 (69.9\%) patients. In 43 of the remaining 65 cases, the patients presented at least 1 year after the initial endoscopy at our hospital without gastrointestinal complaints and without the need for another endoscopy being performed. These cases were therefore considered benign. The other 22 patients were not present at our hospital more than 1 year after the ulcer had first been noted. These patients were also considered as having benign ulcers because in none of them was gastric malignancy registered in our national pathologic database (PALGA). In Fig. 2 we present a flow chart of the 321 patients with a gastric ulcer with comparison of the endoscopic impression and definite histologic diagnosis.

Benign-appearing gastric ulcers at first endoscopy

In 200 patients the ulcer was classified as benign appearing at the first endoscopy. However, in five of these patients $(2.5 \%)$ the ulcer was proven malignant pathologically. In all of these five neoplasms the biopsies taken at the index gastroscopy revealed a non-benign histology. Malignancy 
Fig. 1 Examples of patients with benign and malignant ulcers. a A 3-cm-large ulcer at the lesser curvature. The ulcer is clean based, has no elevated border and is symmetrical. At follow-up endoscopy 4 weeks later a scar is visible and biopsy confirmed no malignancy. b A 7-mm ulcer located in the antrum. The ulcer is clean based, shows no elevated borders and has regular borders. Histology showed no malignancy and healing was confirmed during follow-up endoscopy. c A 15-mm ulcer located at the angulus with a slightly raised border. This ulcus is clean based but has an irregular border. The

endoscopic report contained an explicit suspicion of malignancy. The histology demonstrated adenocarcinoma. d An ulcer of $3 \mathrm{~cm}$ diameter located at the lesser curvature. The ulcer is dirty based, has an elevated border and is irregular. The histology confirmed the suspicion of malignancy
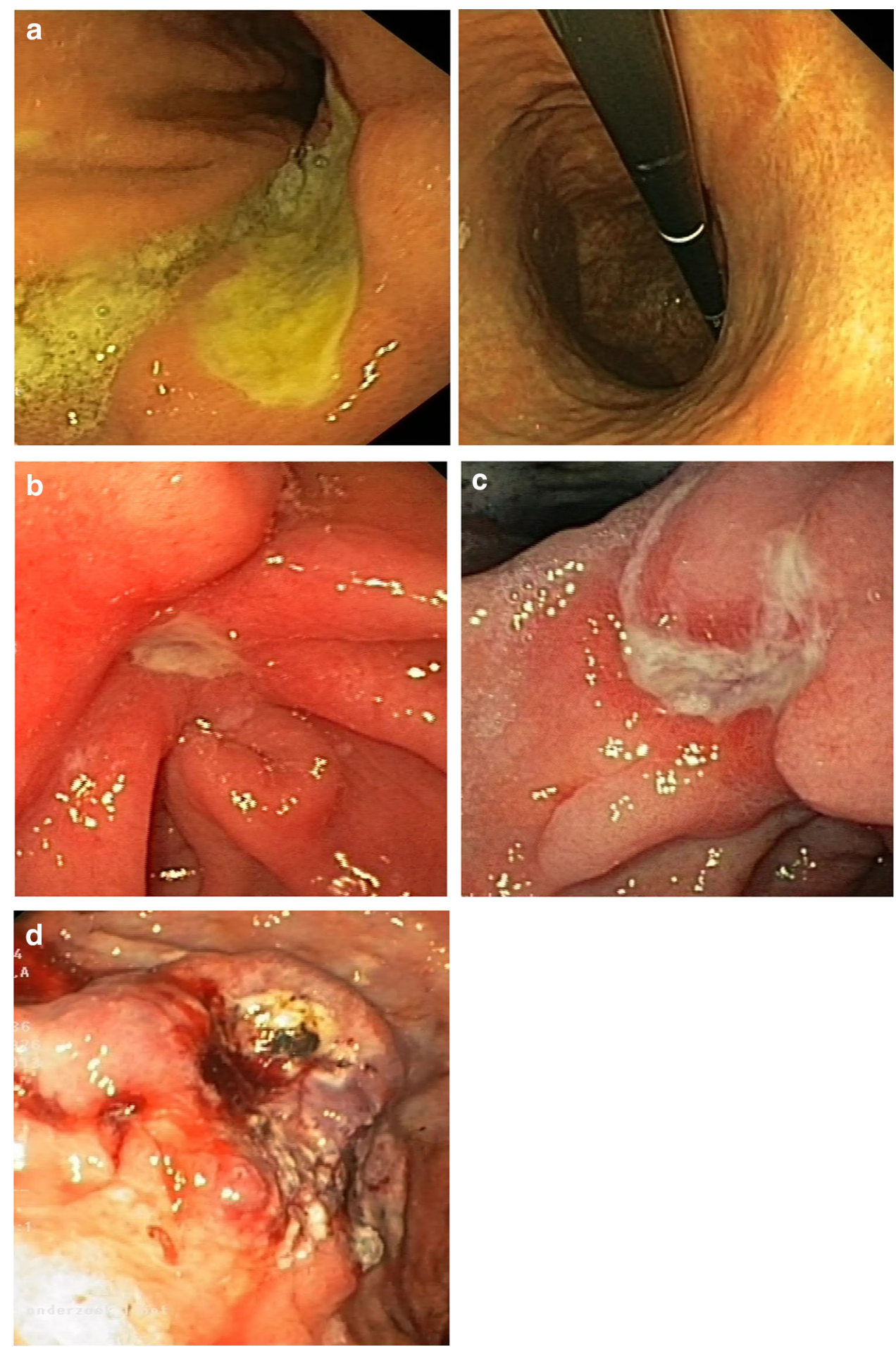

was confirmed first by histology in four cases and in the other case the biopsies of the index endoscopy revealed severe dysplasia.

The accuracy of endoscopic malignancy diagnosis was as follows: sensitivity of 0.98 and specificity 0.84 , positive predictive value 0.84 and negative predictive value 0.98 .
Malignant-appearing gastric ulcers at first endoscopy

In 121 patients the ulcers were explicitly labelled as potentially malignant in the report of the first endoscopy. Of these 121 ulcers, $102(84.3 \%)$ were indeed malignant as confirmed by histology. The definite histology was 
Table 1 Demographic features of the 321 patients with a gastric ulcer

\begin{tabular}{lll}
\hline & \multicolumn{2}{l}{ Definite histologic diagnosis } \\
\cline { 2 - 3 } & Benign & Malignant \\
\hline Number of cases & 214 & 107 \\
Gender (M/F) & $116 / 98(54.2 /$ & $59 / 48(55.1 /$ \\
& $45.8 \%)$ & $44.9 \%)$ \\
Age, median (range), in & $68.8(22-95)$ & $73.4(30-94)$ \\
$\quad$ years & & \\
\hline
\end{tabular}

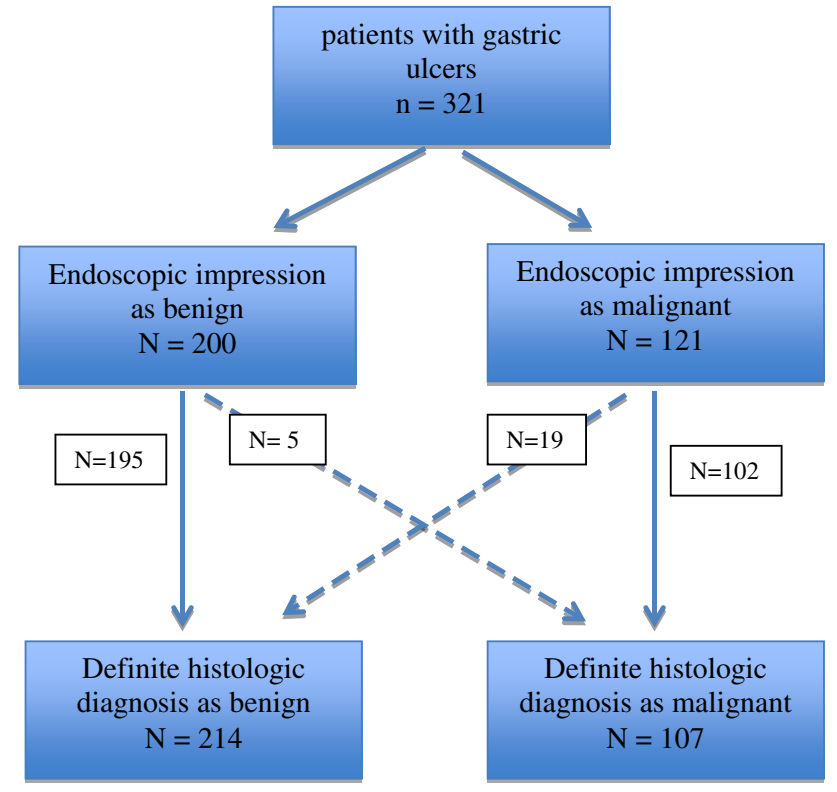

Fig. 2 Flow chart of the patients with a gastric ulcer with comparison of the endoscopic impression and definite histologic diagnosis

intestinal type adenocarcinoma in 71 patients $(70.3 \%)$, diffuse type adenocarcinoma in 13 cases $(12.9 \%)$ and lymphomas in 14 patients (13.9\%); in 2 patients the ulcer was a gastrointestinal stromal tumour and 1 malignant ulcer was a metastasis of breast cancer. The TNM stage of the 84 patients with an adenocarcinoma is shown in Table 2.

In ten patients the ulcer was labelled as potentially malignant according to its appearance by the endoscopist but the malignant histology had not yet been confirmed at the index endoscopy. Therefore, the sensitivity of a benign appearance of the ulcer in combination with benign histology at the first endoscopy is $100 \%$ to rule out malignancy.

The sensitivity of the three potential malignant characteristics of the ulcer at endoscopy were dirty base $79 \%$, elevated border $94 \%$ and irregular border $91 \%$. The specificity of these characteristics was 93,82 and $89 \%$, respectively.
Table 2 Stage of cancer at diagnosis in 84 patients with adenocarcinoma according to the TNM classification

\begin{tabular}{lc}
\hline $\begin{array}{l}\text { Stage of gastric } \\
\text { cancer }\end{array}$ & $\begin{array}{l}\text { Total } \\
(n=84)\end{array}$ \\
\hline IA & $4(5.3 \%)$ \\
IB & $7(9.2 \%)$ \\
II & $9(11.8 \%)$ \\
IIIA & $8(10.5 \%)$ \\
IIIB & $4(5.3 \%)$ \\
IV & $44(57.9 \%)$ \\
Unknown & $8(9.5 \%)$ \\
\hline
\end{tabular}

Table 3 Distribution of the region and the aetiology of the gastric ulcers in 321 patients

\begin{tabular}{lccc}
\hline Location & Total $n(\%)^{\mathrm{b}}$ & Malignant $n(\%)^{\mathrm{c}}$ & Benign $n(\%)^{\mathrm{c}}$ \\
\hline Antrum & $178(55.5)$ & $32(18.0)$ & $146(82.0)$ \\
Angulus & $28(8.7)$ & $8(28.6)$ & $20(71.4)$ \\
Corpus & $55(17.1)$ & $25(45.5)$ & $30(54.5)$ \\
Fundus & $12(3.7)$ & $3(25)$ & $9(75)$ \\
Cardia & $46(14.3)$ & $37(80.4)$ & $9(19.6)$ \\
Other $^{\mathrm{a}}$ & $2(0.6)$ & $1(50)$ & $1(50)$ \\
\hline
\end{tabular}

${ }^{\text {a }}$ Ulcer located at the anastomosis after partial stomach resection

b The percentage in the 'total' column represents the proportional distribution of each localisation

c The percentage under the 'malignant' and 'benign' column represents the proportion of malignant respectively benign aetiology for each localisation

Location and size of the gastric ulcers

The location of the ulcers is described in Table 3. Most of the gastric ulcers were located in the antrum (55.5\%). The antrum was also the predominant location of benign ulcers. Only $18 \%$ of the ulcers located in the antrum were malignant. Markedly, $82.6 \%$ of the ulcers located in the cardia and $45 \%$ of the ulcers in the corpus were malignant. The distribution of the benign or malignant aetiology of the ulcers is not equal, as shown in Table 3.

The size of the ulcers is reported in Table 4. Of all gastric ulcers, $40.3 \%$ were smaller than $1 \mathrm{~cm}$ in size and in $98 \%$ of these patients the ulcers were classified as benign. In contrast, $30.5 \%$ of the patients had an ulcer of more than $3 \mathrm{~cm}$ and the rate of malignancy in this group was as high as $86 \%$. The size of the ulcers was significantly higher in the malignant group compared to the benign group $(p<0.0001)$.

Medication use

The medication use of the patients is shown in Table 5 . NSAIDs were more used among the patients with benign lesions. 
Table 4 Comparison of the size of the ulcer in 318 patients with benign or malignant gastric ulcers

\begin{tabular}{lllc}
\hline $\begin{array}{l}\text { Size (in } \\
\text { diameter })\end{array}$ & $\begin{array}{l}\text { Total } \\
(n=318)^{\mathrm{a}}\end{array}$ & $\begin{array}{l}\text { Benign }^{\mathrm{b}} \\
(n=212)\end{array}$ & $\begin{array}{l}\text { Malignant }^{\mathrm{b}} \\
(n=106)\end{array}$ \\
\hline$<1 \mathrm{~cm}$ & 128 & $125(97.7 \%)$ & $3(2.3 \%)$ \\
$1-3 \mathrm{~cm}$ & 93 & $73(78.5 \%)$ & $20(21.5 \%)$ \\
$>3 \mathrm{~cm}$ & 97 & $14(14.4 \%)$ & $83(85.6 \%)$ \\
\hline
\end{tabular}

${ }^{a}$ In three cases ( 1 malignant and 2 benign) the size of the ulcer was not specifically noted in the endoscopy report

b The percentage represents the proportion of benign or malignant aetiology for each ulcer size

Table 5 Medication use and Helicobacter pylori infection in 321 patients with a gastric ulcer

\begin{tabular}{lccc}
\hline & $\begin{array}{l}\text { Total } \\
(n=321)\end{array}$ & $\begin{array}{l}\text { Benign } \\
(n=214)\end{array}$ & $\begin{array}{l}\text { Malignant } \\
(n=107)\end{array}$ \\
\hline $\begin{array}{l}\text { Aspirin } \\
\text { Yes }\end{array}$ & 83 & $57(26.6 \%)$ & $26(24.3 \%)$ \\
No & 184 & $120(56.1 \%)$ & $64(59.8 \%)$ \\
Missing & 54 & $37(17.3 \%)$ & $17(15.9 \%)$ \\
NSAIDs & & $51(23.8 \%)$ & $9(8.4 \%)$ \\
Yes & 60 & $125(58.4 \%)$ & $81(75.7 \%)$ \\
No & 206 & $38(17.8 \%)$ & $17(15.9 \%)$ \\
Missing & 55 & & $15(14.0 \%)$ \\
Helicobacter pylori & $99(46.3 \%)$ & $92(86.0 \%)$ \\
Yes & 114 & $99(46.3 \%)$ & $0(0 \%)$ \\
No & 191 & $16(7.5 \%)$ & \\
Missing & 16 & &
\end{tabular}

Reducing health costs by not performing follow-up endoscopy

In total 546 gastroscopies were performed in 321 patients with a gastric ulcer, of which 225 were follow-up endoscopies with a maximum of 7 gastroscopies to confirm healing in 2 patients. By not monitoring ulcers considered benign in both endoscopic appearance and histology, 173 gastroscopies would not have been performed, resulting in a reduction of $77 \%$ of follow-up endoscopies. In our hospital, the costs of a gastroscopy are approximately $€ 400$. Therefore, potentially almost $€ 70,000$ could have been saved by not performing follow-up endoscopy during the 6-year study period.

\section{Discussion}

The aim of this study was to assess the diagnostic accuracy of endoscopic follow-up for gastric ulcers. We were driven by the question of whether there is a need to perform follow-up endoscopy in case of a benign-appearing ulcer with benign histology. This study demonstrated that surveillance endoscopy was not needed in our population in case of gastric ulcers both considered benign by appearance and with benign histology at the first endoscopy because it did not reveal an unsuspected malignancy during follow-up.

The sensitivity and specificity of the potential malignant endoscopic characteristics were high. The sensitivity of endoscopic suspicion in combination with histologic examination at the first endoscopy was $100 \%$, which is in line with the $99 \%$ accuracy of endoscopic characteristics in combination with histologic analysis found by others [11, 17]. Also, Manas et al. [9] discovered only one malignancy among the 279 second-look endoscopies performed in their prospective study. Bustamante et al. [18] confirmed the highly accurate endoscopic impression concerning the nature of gastric ulcers. In this retrospective study histology was taken from 341 ulcers. The endoscopic impression compared to the histologic diagnosis showed the following results: positive predictive value 0.68 , negative predictive value 0.98 , sensitivity 0.82 and specificity 0.95 . The endoscopist initially had an uncertain opinion in all three cases in which the first pathology report was benign but nevertheless malignant in repeat histology.

We are aware that the three characteristics used to describe the ulcers are subjective and during the study period, for example, chromoendoscopy or magnifying narrow band imaging in combination with conventional white light imaging was not used routinely to determine the ulcer further. Both image-enhanced endoscopic techniques have been proven to be more accurate in conjunction with C-WLI than only using C-WLI in the diagnosis of early gastric cancer [19, 20].

In our study we found a relatively high proportion of malignant ulcers (33\%) compared to $8-12 \%$ in other studies $[9,18]$. A possible explanation for the high proportion of malignancy in our study is that the ulcers that were not suspicious for malignancy were not registered using the key word "gastric ulcer" in ENDOBASE but were labelled as "erosive gastritis", for example. However, we assume this does not negatively influence our study results because no malignant cases would have been missed. This could underline even more the accuracy of the evaluation made by the endoscopist.

The size of the ulcer and location in the stomach were associated with a high risk of malignancy. This study underlines that ulcers bigger than $3 \mathrm{~cm}$ usually have a malignant origin. We also showed that in most cases ulcers located in the cardia, and to a lesser extent in the corpus, were malignant neoplasms. Our results are in line with the study by Koçak et al. [21]. In this retrospective study 278 patients with a gastric ulcer were analysed. The highest risk of malignancy was related to localisation in the cardia (74\%) and the corpus (54.2\%). Also, in this study the 
likelihood of malignancy of gastric ulcers was closely related to the tumour size ranging from a malignancy percentage of $1.2 \%$ in ulcers smaller than $1 \mathrm{~cm}$ to $72.5 \%$ in ulcers bigger than $5 \mathrm{~cm}$. Apart from the known endoscopic features of malignancy such as a dirty base, elevated or irregular border, the endoscopist should therefore also take the size and location of the ulcer in consideration when determining if an ulcer is potentially malignant.

The need for adequate biopsies is well known. Graham et al. [15] demonstrated in a prospective study that the yield of correct diagnosis in case of gastric cancer increases from 70 to $95 \%$ if four biopsy specimens are taken instead of only one. In our institution multiple biopsies are usually taken from the edge and centre of the ulcer. Because of this adequate biopsy protocol all of the five benign-appearing neoplasms at the first endoscopy that were ultimately malignant were not missed and were eventually well classified.

NSAIDs, including aspirin, increase the risk of developing gastroduodenal ulcers. The risk of ulcer as a complication amongst NSAID users has been estimated to be between 25 and $35 \%$ [22]. In our study, as expected, the use of NSAIDs was higher amongst the cases with benign ulcers compared to malignant ulcers. Interestingly, there was no difference in the use of aspirin between the malignant and benign subgroups of patients.

Strong aspects of our study are the large number of patients included, long follow-up in the study period and strict patient selection. Also, the chance of falsely labeling an ulcer as benign was minimised by the PALGA search in the national pathology database for possible gastric malignancy detected in other hospitals. All endoscopies were conducted in the same hospital, which used a strict biopsy protocol for gastric ulcers.

A minor drawback of this study is its retrospective design. However, we could retrieve almost all the important information because we used an automated endoscopy documentation system that includes standard text blocks to describe size and other characteristics of the ulcer, for example.

In conclusion, we consider that surveillance endoscopy should be individualised instead of being the standard of care. This study suggests that surveillance is only needed for malignant-appearing ulcers with benign histology at the first endoscopy, especially if the ulcer is located at the cardia or corpus, or is bigger than $3 \mathrm{~cm}$. Also, if histology is suspicious but not yet definite for malignancy, the patient needs endoscopic surveillance with repeat biopsy from the edge and centre of the ulcer.

Abandoning the current guidelines and not performing follow-up endoscopy in patients with benign-appearing ulcers and no dysplasia in histology would save the patients a lot of distress and could reduce hospital costs. However, before abandoning the current guidelines a prospective study with larger numbers of patients is needed to further minimise the small risk of missing gastric cancer by not performing surveillance endoscopy.

\section{References}

1. Everhart JE, Byrd-Holt D, Sonnenberg A. Incidence and risk factors for self-reported peptic ulcer disease in the United States. Am J Epidemiol. 1998 Mar 15;147(6):529-36.

2. Multidisciplinaire Richtlijn maagklachten 2004, kwaliteitsinstituut voor de Gezondheidszorg CBO. Dutch guideline of gastroenterology and hepatology.

3. Dyspepsia management guidelines 2004, Britisch Society of Gastroenterology.

4. Indication à visée diagnostique de l'endoscopie digestive haute en pathologie oeso-gastro-duodénale de l'adulte à l'exclusion de l'echoendoscopie et l'enteroscopie 2001. Agence Nationale d'Accreditation et d'évaluation en santé.

5. Sakita T, Oguro Y, Takasu S, Fukutomi H. Observations on the healing of ulceration in early gastric cancer. The life cycle of malignant ulcer. Gastroenterology. 1971;60:835.

6. Paulino F, Roselli A. Early gastric cancer: report of 25 cases. Surgery. 1979;85:171-6.

7. Moutford RA, Brown P, Salmon PR, Alvarenga C, Neumann CS, Read AE. Gastric cancer detection in gastric ulcer disease. Gut. 1980;21:9-17.

8. Lupano F, Sategna-Guidetti C. Endoscopic follow-up of patients with gastric ulcer. Prospect Study. J. Clin Gastroenterol. 1986 Aug;8(4):430-4.

9. Mañas MD, Domper A, Albillos A, Hernández A, Carpintero P, Lorente R, et al. Endoscopic follow-up of gastric ulcer in a population at intermediate risk for gastric cancer. Rev Esp Enferm Dig. 2009 May;101(5):317-24.

10. Pruitt RE, Truss CD. Endoscopy, gastric ulcer, and gastric cancer. Follow-up endoscopy for all gastric ulcers? Dig Dis Sci. 1993 Feb;38(2):284-8.

11. Bytzer P. Endoscopic follow-up study of gastric ulcer to detect malignancy: is it worthwhile? Scand J Gastroenterol. 1991 Nov;26(11):1193-9.

12. Hopper AN, Stephens MR, Lewis WG, Blackshaw GR, Morgan MA, Thompson I, et al. Relative value of repeat gastric ulcer surveillance gastroscopy in diagnosing gastric cancer. Gastric Cancer. 2006;9(3):217-22.

13. Eckardt VF, Giessler W, Kanzler G, Bernhard G. Does endoscopic follow-up improve the outcome of patients with benign gastric ulcers and gastric cancer? Cancer. 1992;69:301-5.

14. Yeh Ho, Hur Chun. Cost-effectiveness of endoscopic surveillance of gastric ulcers to improve survival. Gastrointest Endosc. 2010 Jul;72(1):33-43.

15. Graham DY, Schwartz JT, Cain GD, Gyorkey F. Prospective evaluation of biopsy number in the diagnosis of esophageal and gastric carcinoma. Gastroenterology. 1982;82:228-31.

16. Stolte M, Seitter V, Müller H. Improvement in the quality of the endoscopic/bioptic diagnosis of gastric ulcers between 1990 and 1997- an analysis of 1,658 patients. Z Gastroenterol. 2001 May;39(5):349-55.

17. Llanos O, Guzmán S, Duarte I. Accuracy of the first endoscopic procedure in the differential diagnosis of gastric lesions. Ann Surg. 1982 Feb;195(2):224-6.

18. Bustamante M, Devesa F, Borghol A, Ortuño J, Ferrando MJ. Accuracy of the initial endoscopic diagnosis in the discrimination 
of gastriculcers: is endoscopic follow-up study always needed? J Clin Gastroenterol. 2002 Jul;35(1):25-8.

19. Ezoe Y, Muto M, Uedo N, Doyama H, Yao K, Oda I, et al. Magnifying narrowband imaging is more accurate than conventional white-light imaging in diagnosis of gastric mucosal cancer. Gastroenterology. 2011 Dec;141(6):2017-25.

20. Fujiwara S, Yao K, Nagahama T, Uchita K, Kanemitsu T, Tsurumi $\mathrm{K}$ et al. Can we accurately diagnose minute gastric cancers ( $\leq 5 \mathrm{~mm})$ ? chromoendoscopy (CE) vs magnifying endoscopy with narrow band imaging (M-NBI). Gastric Cancer. 2014 Jul 9.
21. Koçak E, Kılıç F, Akbal E, Taş A, Köklü S, Filik L, Bıyıkoğlu I, Ergül B. The usefulness of ulcersizeandlocation in the differential diagnosis of benign and malignant gastric ulcer. Wien Klin Wochenschr. 2013 Jan;125(1-2):21-5.

22. Hawkey CJ. Nonsteroidal anti-inflammatory drug gastropathy. Gastroenterology. 2000 Aug;119(2):521-35. 